\title{
Heat Capacity of Silver Iodide. V. Heat Content of Nonstoichiometric Material
}

\author{
C. M. Perroti* and N. H. Fletcher \\ Department of Physics, University of New England, Armidale N.S.W., Australia
}

(Received 12 November 1969)

\begin{abstract}
A program of study of the cation disorder in $\alpha$-AgI is completed by a series of heat content experiments on quenched samples which contain small excesses of silver. The results presented are analyzed in terms of lattice disorder, and a model of the behavior of doped material is developed. This model shows that despite significant differences in thermal properties, the cooperative disordering of the cations is very similar in stoichiometric and doped material. It is proposed that deviation from stoichiometry causes an elevation of the critical temperature for disordering.
\end{abstract}

\section{INTRODUCTION}

In the other Papers of this series ${ }^{1-4}$ to be referred to as I, II, III, and IV, we presented data showing that the phase of silver iodide called $\alpha$-AgI (stable between $150^{\circ} \mathrm{C}$ and the melting point $555^{\circ} \mathrm{C}$ ) undergoes a rather complex order-disorder transition during heating. This transformation has been identified from the anomalous heat capacity of annealed $\alpha$-AgI and also from heat content experiments on nominally pure material quenched from a high temperature. Our physical model of the material is based on the model proposed by Strock $^{5}$ on the basis of $x$-ray powder diffraction experiments and is briefly as follows: The iodide ions occupy the sites of a regular bcc array and the two silver ions are distributed over 42 possible positions in the unit cell (Fig. 1). The distribution of cations is not random but displays correlations between the positioning of ions, these correlations being expressible in terms of three interpenetrating sublattices for the silver ions. Each sublattice is bcc with the same lattice parameter as the bcc lattice of iodide ions and the distribution of cations can be expressed adequately in terms of the over-all occupation probabilities of the sublattices, one having occupation probability $\sigma$ and the other two having occupation probability $S=(1-\sigma) / 2$. As the temperature is increased, the cations disorder progressively and $\sigma$ decreases from unity at very low temperatures to progressively smaller values. At the critical temperature, $430^{\circ} \mathrm{C}$, the disordering is suddenly completed and $\sigma$ changes discontinuously to the value $\frac{1}{3}$ which represents the fully disordered state existing at all temperatures above $430^{\circ} \mathrm{C}$.

When the heat capacity of annealed samples which had been doped with small excesses of silver or iodine was measured, no evidence of this order-disorder transition was found, $C_{v}$ being constant at $\sim 12 \mathrm{cal} / \mathrm{mole} \cdot \mathrm{deg}$ over the entire temperature interval in which $\alpha$-AgI is stable. Within the range used, 0.5-2.5 mole $\%$, this effect did not seem to depend on the doping level and, since complete order in the material is most unlikely, we noted that the behavior seemed consistent with the assumption that the deviation from stoichiometry de- creased the critical temperature to below the $150^{\circ} \mathrm{C}$ phase transformation in the material.

This explanation supposes extreme sensitivity of the interaction energies within the material to composition and is therefore rather disturbing. In addition, it requires a significant increase in the latent heat of transformation at $150^{\circ} \mathrm{C}$ as a result of doping. No change was actually observed. The purpose of the present paper is therefore to present the results of heat content content experiments on doped material and, after showing the vast differences between them and the results for nominally pure material ${ }^{4}$ (Paper IV), to develop a more satisfactory explanation of the effects of doping.

\section{HEAT CONTENT EXPERIMENTS}

\section{A. Quenching Results}

Experiments were performed in which samples doped with various amounts of silver were placed in a furnace and annealed for a set time before being water-quenched to room temperature, their heat capacity being subsequently measured on reheating. The results were characterized by plotting curves of $\delta C_{p}$, the amount by which the heat capacity increased as a result of quenching, and showed no sensitivity to doping level. A typical $\delta C_{p}$ curve (a of Fig. 2) shows an extensive energy absorption in the temperature region appropriate to $\alpha$-AgI.

Analyzing the effects of various heat treatments in the same manner as was described in Paper IV, we have established that two separate absorption processes accompany the annealing of quenched nonstoichiometric samples. There is a sharp absorption peak (labeled II) superimposed on an extended energy absorption (labeled I). The peak II is very similar to that observed with quenched stoichiometric material, while the dominant effect (I) proceeds under a diffusioncontrolled monomolecular mechanism ${ }^{4}$ in the same way as did the dominant effect observed for stoichiometric material. As a point of difference, however, the latter case featured extensive energy evolution rather than absorption. Again proceeding in the manner previously described, ${ }^{4}$ we have established that the nonequilib- 


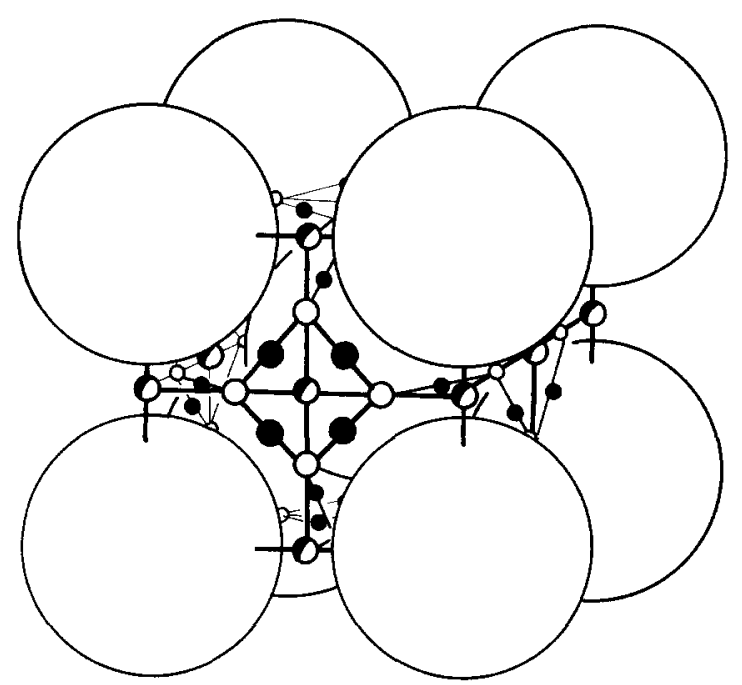

FIG. 1. Schematic drawing of the structure of $\alpha$-AgI. The large spheres represent iodide ions and the remaining sites are available to the silver ions.

rium effects shown in Fig. 2(a) are caused by the rapid cooling of the $\alpha$ phase rather than by rapid traversal of the phase transformation at $150^{\circ} \mathrm{C}$. Since these effects would therefore be expected for a hypothetical quench to $160^{\circ} \mathrm{C}$ (say), it seems reasonable to assume that their occurrence can be explained in terms of the temperature dependence of the equilibrium state of $\alpha$-AgI.

The amounts of energy associated with the two processes depend on quenching temperature in the manner of Fig. 3 in which it is also shown that the latent heat of transformation at $150^{\circ} \mathrm{C}$ is always significantly lower than the usual value $1.5 \mathrm{kcal} / \mathrm{mole}$ and does not vary with quenching temperature.

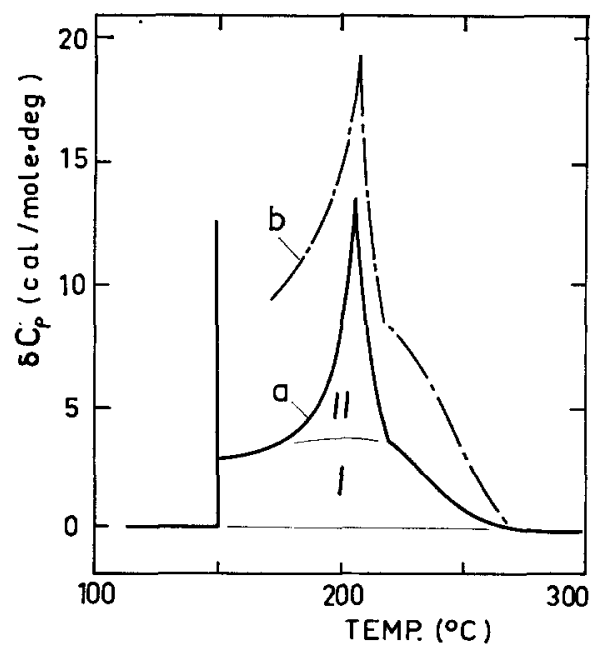

FIG. 2. Heat capacity deviation in silver-doped AgI: (a) initial heating after water-quenching from $350^{\circ} \mathrm{C}$. note the two energy absorption processes (I and II) ]; (b) immediate reheating after cooling from 350 to $160^{\circ} \mathrm{C}$ at $20 \mathrm{deg} / \mathrm{min}$.

\section{B. Discussion of Quenching Results}

The sharp absorption process II and the depression of the latent heat of transformation at $150^{\circ} \mathrm{C}$ were both observed with nominally pure samples and no doubt have the same explanations, although we do not understand the effects at present. The dominant feature of the results for pure samples was an extensive energy release, and we saw that this could be explained as the relaxation of excessive cation disorder retained on quenching. With doped samples, this effect has apparently become an extensive energy absorption and although we would like to attribute its occurrence to some similar process, the reversed sign of the effect cannot be explained easily. In fact, the usual defects retained during a quench all give rise to energy release, and there is no analogy which we can draw in order to explain this effect.

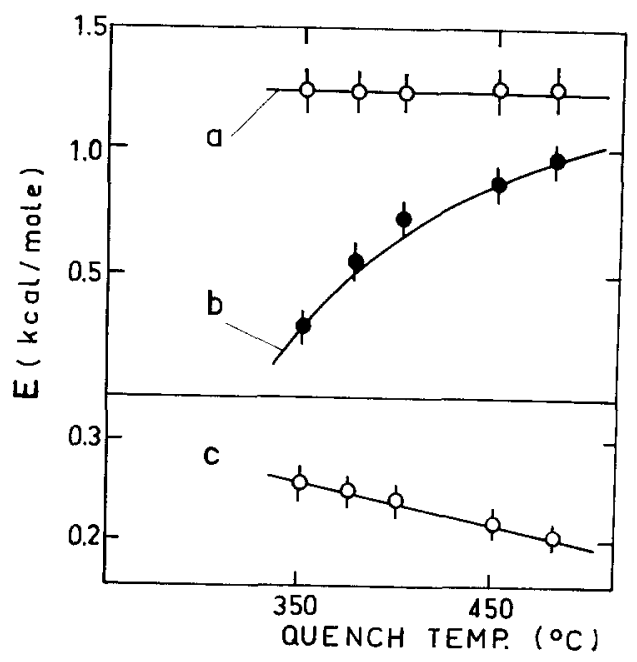

FIG. 3. Energy associated with nonequilibrium effects in waterquenched silver-doped AgI: (a) latent heat of transformation at $150^{\circ} \mathrm{C}$; (b) energy absorption process $\mathrm{I}$; (c) energy absorption process II.

If it were correct to assume that doped $\alpha$ - $\mathrm{AgI}$ is always fully disordered, the cation disorder would not be temperature dependent and nonequilibrium effects involving this disorder would not be observed after quenching. The only effect which could cause energy absorption would be the retention of extra silver in the structure, it being assumed that the doping levels were high enough to ensure that the deviation from stoichiometry was at its maximum value at all temperatures and therefore increased with increasing temperature. From the discussion in the first paper of this series, ${ }^{1}$ we find that for the doping levels used in these experiments the maximum excess of silver in the structure at the melting point was 0.5 mole $\%$. If retained during a quench, this silver would be expelled on reheating and 
the process would require the absorption of $0.15 \mathrm{kcal}$ by a mole of quenched sample. Since this is just not comparable with the observed effect, the explanation cannot be applicable. In fact, the variation of the magnitudes of the absorption processes I and II (Fig. 3) also cannot be explained adequately on the basis of this model of doped material. Note, however, that the curves of Fig. 3 do not have plateaus, and there is no reason to suggest that a critical temperature occurs within the range $150-555^{\circ} \mathrm{C}$. Any model proposed for the material must predict that the critical temperature falls outside this range, and since we have shown that it cannot be below $150^{\circ} \mathrm{C}$, the only remaining possibility is that the deviation from stoichiometry causes an elevation of the critical temperature.

Before attempting to develop an appropriate model, however, let us look at some of the other results which we will need to be able to explain.

\section{Other Heat Content Results}

The $\delta C_{p}$ curves obtained after a silver-doped sample was cooled to room temperature at a rate of $\sim 20 \mathrm{deg} /$ min were very similar to those obtained after quenching. A typical curve is shown in Fig. 2 (b), and it is seen that the magnitude of the absorption II is unchanged while that of absorption $\mathrm{I}$ is significantly greater than for the corresponding quench. The depression of the latent heat of transformation at $150^{\circ} \mathrm{C}$ was the same for both cases.

The heat content of iodine-doped samples was also studied, and the results obtained were very similar to those obtained for silver-doped samples.

\section{THEORY FOR NONSTOICHIOMETRIC MATERIAL}

Turning to the problem of developing a reasonable model for doped $\alpha$-AgI, we need to introduce some parameter into our existing theory ${ }^{2,3}$ (Papers II and III) in order to specify the actual amount of doping agent incorporated in the structure. This parameter, which we will call $x$, will measure the number of excess silver ions in mole percent and is introduced into the theory by means of the requirement that the total number of occupied sites on the three bcc sublattices available to silver ions must be exactly equal to the total number of silver ions. Mathematically, this becomes

$$
\sigma+2 S=1+x / 100
$$

which replaces the relationship between sublattice occupation probabilities $(\sigma+2 S=1)$ used in the previous papers. Positive values of $x$ represent silver excess and negative values represent silver deficiency (iodine excess).

For small deviations from stoichiometry, the predictions of our theory are nearly the same as for the stoichiometric case. In all cases, the cations are preferentially ordered onto one of the three sublattices at low temperatures and cooperatively disorder during heating until the critical temperature is reached and the disordering is suddenly completed. The critical temperature is always associated with a latent heat and always occurs near the value of reduced temperature $\tau=0.9$.

We have already decided that the critical temperature $T_{c}$ of doped material must fall above the melting point and, in order to explain the elevation of critical temperature, we have to look at the definition of reduced temperature,

$$
\tau \equiv k T / v \text {. }
$$

Since $\tau_{c}$ does not change appreciably, doping can only shift $T_{c}$ to higher values if it causes an increase in the ordering energy $v$. Is this possible?

The interactions which specify the ordering energy of the silver ion distribution are those between the sites (whether occupied or vacant) of different cation sublattices. Since we based our cooperative model ${ }^{2}$ of $\alpha$-AgI on the notion of stress interactions transmitted by the iodide ion lattice which suffers severe distortions in order to accommodate the cations, it is reasonable to assume that changes in the strengths of these interactions will accompany changes in the composition of the material. That the interaction energies should be increased by deviation from stoichiometry would seem to be supported by our own observations during the course of crystal growth experiments: Crystals containing as little as $0.3 \%$ of impurity are significantly harder than high-purity crystals. Some of this effect may of course be due to the formation of microprecipitate particles, although one would not expect this to occur in the growth method used, but a genuine bulk effect is not unlikely.

These interactions will depend on cation concentration in such a way that if one interaction energy is changed, all others will change by the same factor. We shall call this presently unknown factor $\chi(x)$, and it may be expressed in the form

$$
\chi(x)=v_{A A}(x) / v_{A A^{0}}=\cdots=v(x) / v^{0},
$$

where $-v_{A A}(x), \ldots$ and $-v_{A A}{ }^{0}, \ldots$ are, respectively, the values of the interaction energies $-v_{A A}, \ldots$ at composition $x$ and at stoichiometry.

Although we cannot deduce the form of $\chi(x)$ without great difficulty, we can see whether it is possible to use these ideas to explain our results. The samples which we used were doped with sufficient silver or iodine that it is almost certain that all of the doping agent was never included in the structure. In this case, the composition of the silver-rich samples, for example, would depend only on temperature and not on the doping levels. Thus, their behaviors would be expected to be the same, as observed. For all of the samples used, the 


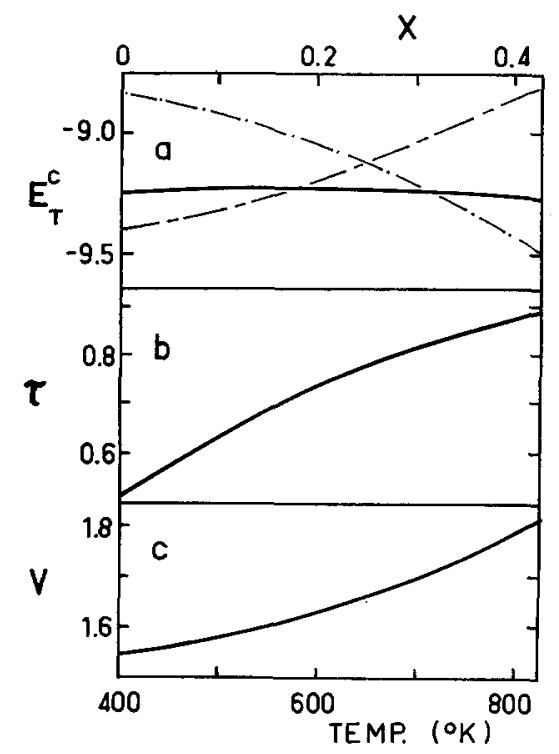

Fig. 4. Temperature and composition dependence of: (a) configurational energy (in $\mathrm{kcal} / \mathrm{mole})\left[E_{T^{c}},-\right.$; $\left(E^{c}-3.5\right),---$; $(-\xi c-5.5),-\cdots]$; (b) reduced temperature; (c) ordering energy (in $\mathrm{kcal} / \mathrm{mole}$ ) of the cation distribution in silver-doped $\alpha$-AgI, according to the model discussed in the text.

deviation from stoichiometry can therefore be assumed to increase with increasing temperature and, if we assume that $\chi(x)$ increases as $|x|$ increases, the various interaction energies also increase with increasing temperature.

Assuming that we knew the actual variation of composition and the function $\chi(x)$, we could now calculate the temperature dependence of the configurational energy of the doped samples. This, however, is not the energy $E^{c}(\tau, x)$ which is calculated by the procedure outlined in Paper II in this series. ${ }^{2}$ In that paper, we simplified the algebra by shifting the zero of configurational energy by a constant amount. Under these conditions, the shift of the zero is no longer constant, but varies with $x$ and, hence, with temperature. The true configurational energy of the cations is

where

$$
E_{T^{c}}(\tau, x)=E^{c}(\tau, x)-\xi^{c}(x),
$$

$$
\begin{aligned}
\xi^{c}(x) & \equiv 2 N\left[3 v_{B B}^{0}+\left(v_{A A}^{0}-v^{0}{ }_{B B}\right)(1+x / 100)\right] \chi(x) \\
& \approx 2 N\left(2 v^{0}{ }_{B B}+v^{0}{ }_{A A}\right) \chi(x) .
\end{aligned}
$$

Since we do not have the required information, the only way in which we can demonstrate the method of solution and the results which may be expected is by adopting a reasonable physical model. Doing this, we will consider silver-rich material and assume that the variation of composition with temperature is linear, $x$ increasing from zero at $400^{\circ} \mathrm{K}$ to 0.4 at $800^{\circ} \mathrm{K}$. This is well within the limits set by our experiments, and in order to obtain the minimum shift in critical tempera- ture compatible with the experiments, we must assume that the value of $\chi(x)$ at the melting point is sufficiently great to change the ordering energy $v$ of the alloy from the $1.54 \mathrm{kcal} / \mathrm{mole}$ characteristic of stoichiometric material to $1.82 \mathrm{kcal} / \mathrm{mole}$, this setting the critical temperature of the doped material at its melting point $828^{\circ} \mathrm{K}$. In other words, we should choose a form for $\chi(x)$ which satisfies the boundary conditions

$$
\chi(0)=1 \text { and } \chi(0.43)=1.18 .
$$

Such a function is the quadratic

$$
\chi(x)=1+\frac{1}{4} x+\frac{1}{2} x^{2}
$$

and will be adopted because it allows us to predict an almost vanishing configurational heat capacity under our assumed condition of linearly varying composition. Note that the rate of change of composition is too slow for an appreciable amount of energy to be associated with the chemical reaction causing the change.

Using Eqs. (5), (2), and (1), together with the value

$$
v^{0}=1.54 \mathrm{kcal} / \mathrm{mole}
$$

derived from the experiments on nominally pure material, we are now in the position to calculate the variation of ordering energy and reduced temperature as a doped sample is heated. These variations are shown in Fig. 4 and can be used to determine the variation of $E^{c}(\tau, x)$ in Eq. (3). Proceeding in the manner outlined in Paper II, we find that it is possible to plot contour diagrams of the form shown in Fig. 5 which gives the value of $E^{c}(\tau, x) / N v(x)$ for a particular reduced temperature and composition. Using the values thus obtained for the various temperatures in the range $400-$ $800^{\circ} \mathrm{K}$, it is then simply a matter of using the calculated variation of $v(x)$ to obtain $E^{c}(\tau, x)$ as a function of temperature. From the slope of the curve thus obtained [Fig. 4(a)], it can be seen that the contribution to the configurational heat capacity from the progressive disordering of the cations is small and rises from $1 \mathrm{cal} /$ mole $\cdot$ deg just above the $150^{\circ} \mathrm{C}$ phase transformation to $2 \mathrm{cal} / \mathrm{mole} \cdot \mathrm{deg}$ at the melting point.

The second contribution to the configurational heat capacity is also small and arises from the temperature

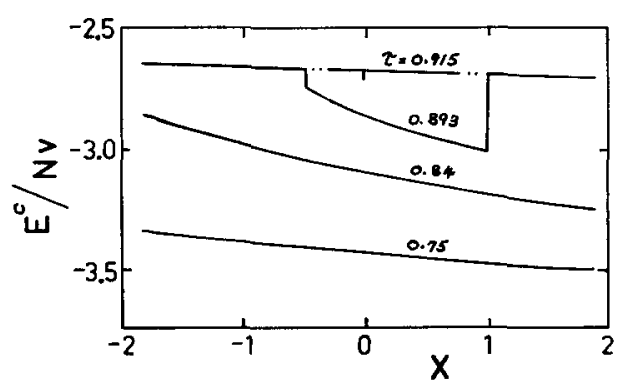

FIG. 5. Contours showing the variation of cation configurational energy $E^{c}$ with reduced temperature and composition. 
dependence of $-\xi^{c}(x)$. Unfortunately, this cannot be calculated exactly until the value of $2 v_{B B}^{0}+v^{0} A A$ is known, but for the particular choice

$$
2 v^{0}{ }_{B B}+v^{0}{ }_{A A}=1.67 \mathrm{kcal} / \mathrm{mole},
$$

the variation of $-\xi^{c}(x)$ is such as to give a total configurational energy $E_{T^{c}}$ which is essentially temperature independent (Fig. 4), meaning that the heat capacity of doped material has no configurational component and will therefore be essentially normal. In fact, the spread of experimental results for doped material is such that any value of $2 v_{B B}^{0}+v_{A A}^{0}$ in the range - 1.5$+3.0 \mathrm{kcal} / \mathrm{mole}$ gives close agreement with experiment, while a much greater range gives results which would be regarded as satisfactory.

We see that the model can easily explain the observations for doped samples. The physical assumptions necessary for this to be possible are apparently quite reasonable and are far from unique. For our model of $x(T)$, the particular form adopted for $\chi(x)$ is the only one which allows the prediction of an almost exactly vanishing configurational heat capacity, but any smoothly varying function gives results well within the experimental limits. Although the model is a gross oversimplification, it clearly demonstrates that the observed heat capacity behavior of annealed material can be explained by assuming that the critical temperature of nonstoichiometric material increases steadily as the deviation from stoichiometry increases. The apparently normal behavior of doped material results from the changing composition and its effect on the energies of interaction within the cation array. It does not, however, indicate that the variation of the degree of cation disorder in doped material is significantly different from that in stoichiometric. Rather, the cations still disorder progressively during heating, as shown by the temperature dependence of the occupation probabilities $\sigma$ and $S$ calculated on the model detailed above and compared with the corresponding curve for stoichiometric material in Fig. 6.

As well as explaining the heat capacity variation of annealed material, the theory shows why there was no change in the latent heat of transformation at $150^{\circ} \mathrm{C}$ as a result of doping. The curves of Fig. 6 show that the configurational energies of nominally pure and doped materials just above the transformation are almost exactly equal, and since the low-temperature phase is not structurally sensitive to small deviations from stoichiometry, this means that the latent heat will be the same for both cases.

Completing the analysis, we now have to seek an explanation of the large absorption process I observed with quenched samples. Returning to the model evaluated above and considering a hypothetical quench from 800 to $450^{\circ} \mathrm{K}$, we see that if all of the disorder and all of the doping agent included in the structure at $800^{\circ} \mathrm{K}$

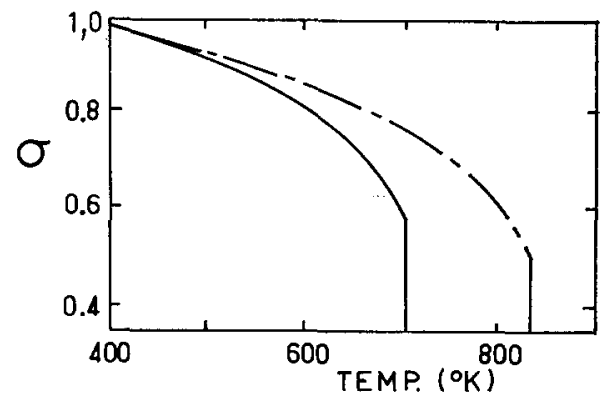

FIG. 6. Variation of the equilibrium values of the order parameter $\sigma$ with absolute temperature in: - , stoichiometric material, and - - - doped material (according to the model discussed in the text).

is retained after the quench, the configurational energy of the quenched sample will be almost the same as that of an annealed sample at $450^{\circ} \mathrm{K}$ (Fig. 4). The only nonequilibrium effect we would expect to observe on heating is the steady absorption of $0.12 \mathrm{kcal} / \mathrm{mole}$ necessary to expel the excess silver in the structure.

Expulsion of the doping agent during quenching requires the transport of silver over macroscopic distances to existing impurity centers or dislocations and is necessarily a slower process than that of cation ordering during quenching. When considering our hypothetical quench, we should have realized that even if all of the doping agent is retained, all of the cation disorder may not be. The extreme case where all of the doping agent and none of the disorder is retained corresponds to a sample with order parameter $\sigma=0.95$ and composition $x=0.4$, this having a total configurational energy which is $1.5 \mathrm{kcal} / \mathrm{mole}$ lower than the equilibrium state and relaxing with a total absorption of $1.6 \mathrm{kcal} / \mathrm{mole}$. The model therefore allows us to explain energy absorptions in the range $0.1-1.6 \mathrm{kcal} / \mathrm{mole}$ by samples quenched from near the melting point. In addition, it predicts that the amount of energy absorbed should increase steadily as the quenching temperature is increased from values near $150^{\circ} \mathrm{C}$ towards the melting point.

We can therefore explain the magnitude and shape of the appropriate curve in Fig. 3 on the basis that a high proportion of the doping agent is retained during quenching but not all of the cation disorder is retained. Accordingly, we might expect that if slower quenches were used in order to retain nearly the same amount of doping agent and much less cation disorder, the energy absorption on reheating should be significantly greater. This effect was noted in the experiments with a cooling rate of $20 \mathrm{deg} / \mathrm{min}$.

\section{CONCLUSION}

It would seem that we have now developed a satisfactory model of the behavior of doped silver iodide in the $\alpha$ phase. This model shows that despite the very different thermal properties, the structural behavior of 
nominally pure and doped samples is very similar and not, as we first thought, unbelievably dissimilar.

This entire investigation of the calorimetric properties of the high-temperature $(\alpha)$ phase of silver iodide has lead to the conclusion that despite differences in composition, samples of this material will always show the same feature which is not included in the structural model developed from previous $x$-ray powder diffraction results, namely a correlated distribution of cations over the available lattice positions. The usual model pictures the cations as randomly distributed over the available positions, predicting no significant change in the degree of disorder during heating. From our results, it is apparent that the cations actually disorder cooperatively during heating.

Since this is a fundamental difference in concept, further investigations of different properties are warranted, the most significant being a new structure determination using single-crystal specimens of $\alpha$-AgI. This, unlike the powder diffraction experiments, would give unequivocal evidence concerning the cation dis- order and its variation with temperature. Thermal studies of the type reported in this series of papers can give no more really new data.

\section{ACKNOWLEDGMENTS}

This program of study of partially disordered solids has been supported financially by the University of New England and by the Australian Research Grants Committee. One of us (C. M. P.) is also grateful to the Commonwealth Scientific and Industrial Research Organization for the award of a Postgraduate Studentship, during the tenure of which this work was done.

* Present address: Division of Pure Physics, National Research Council of Canada, Ottawa, Canada.

${ }_{1}$ C. M. Perrott and N. H. Fletcher, J. Chem. Phys. 48, 2143 (1968)

${ }^{2}$ C. M. Perrott and N. H. Fletcher, J. Chem. Phys. 48, 2681 (1968)

${ }^{3}$ C. M. Perrott and N. H. Fletcher, J. Chem. Phys. 50, 2770 (1969).

${ }^{4}$ C. M. Perrott and N. H. Fletcher, J. Chem. Phys. 52, 3368 (1970), preceding paper.

${ }^{5}$ L. W. Strock, Z. Physik. Chem. B31, 132 (1936).

THE JOURNAL OF CHEMICAL PHYSICS

VOLUME 52, N UMBER 7

1 A P R I L 1970

\title{
Electron Paramagnetic Resonance Studies of a Viscous Nematic Liquid Crystal
}

\author{
G. C. FryburG \\ Lewis Research Center, Cleveland, Ohio 44135 \\ AND \\ E. Geierinter \\ Physics Department and Liquid Crystal Institute, Kent State University, Kent, Ohio
}

(Received 12 November 1969)

\begin{abstract}
We have studied the order as a function of temperature in the liquid crystal bis ( $4^{\prime}-n$-octyloxybenzal) - 2 chloro-1,4-phenylenediamine using vanadyl acetylacetonate as a paramagnetic probe. The order found in the upper part of the mesophase $\left(105-179^{\circ} \mathrm{C}\right)$ was normal. However, in the lower part of the mesophase $\left(40-105^{\circ} \mathrm{C}\right)$ the order found was much higher than ordinarily observed, approaching complete alignment near the melting point. In addition, the EPR lines assumed an unusual shape in this lower temperature region. The observations were accounted for by assuming the liquid crystal was too viscous to allow free tumbling of the probe molecules, and by developing a new model in which a spatial rather than a temporal average was performed. Supporting evidence for the validity of the assumption was obtained experimentally by demonstrating that the liquid crystal had an unusually long response time for reorientation in the magnetic field.
\end{abstract}

\section{INTRODUCTION}

Some years ago Saupe and Englert ${ }^{1}$ showed that liquid crystals can serve as orienting solvents in magnetic resonance studies. Because of the orientational ordering in the mesophase of these solvents, certain anistropic terms in the spin Hamiltonian are not averaged to zero. These anisotropic parameters may be evaluated from an analysis of the spectra obtained. In high-resolution nuclear magnetic resonance spectroscopy the technique has been developed into a method for determining molecular structures. ${ }^{2}$ In electron paramagnetic resonance (EPR) the development has been less spec- tacular. However, one application of EPR has been the determination of the degree of ordering in a nematic liquid crystal in a simple, consistent manner. The technique involves dissolving a small amount (mole fraction $<10^{-3}$ ) of a paramagnetic material in the liquid crystal and measuring the alignment with the magnetic field. With judicious choice of the paramagnetic probe material the alignment of the solute may be related to the order of the liquid crystal.

We were interested in studying the order in the liquid crystal bis $\left(4^{\prime}\right.$ - $n$-octyloxybenzal $)$-2-chloro- 1,4 -phenylenediamine. This liquid crystal was recently synthesized by Arora $e t a l .{ }^{3}$ and is of interest because of its extremely 